\title{
Fast Cooperative Energy Detection under Accuracy Constraints in Cognitive Radio Networks
}

\author{
Shengliang Peng, ${ }^{1,2}$ Weibin Zheng, ${ }^{1}$ Renyang Gao, ${ }^{1}$ and Kejun Lei ${ }^{3}$ \\ ${ }^{1}$ Xiamen Mobile and Multimedia Communications Key Laboratory, Huaqiao University, Xiamen 361021, China \\ ${ }^{2}$ Department of Electrical and Computer Engineering, Stevens Institute of Technology, Hoboken, NJ 07030, USA \\ ${ }^{3}$ College of Information Science and Engineering, Jishou University, Jishou 416000, China
}

Correspondence should be addressed to Shengliang Peng; peng.shengliang@gmail.com

Received 26 May 2017; Accepted 8 August 2017; Published 13 September 2017

Academic Editor: Mohammad Shikh-Bahaei

Copyright (C) 2017 Shengliang Peng et al. This is an open access article distributed under the Creative Commons Attribution License, which permits unrestricted use, distribution, and reproduction in any medium, provided the original work is properly cited.

\begin{abstract}
Cooperative energy detection (CED) is a key technique to identify the spectrum holes in cognitive radio networks. Previous study on this technique mainly aims at improving the detection accuracy, while paying little attention to the performance of detection time. This paper concentrates on the issue of fast CED, which is achieved by minimizing its detection time subject to the constraints on detection accuracy. Firstly, the prevalent counting rule based CED algorithm is optimized. Taking the special cases of counting rule (AND rule and OR rule), for example, we show that detection time can be minimized by selecting an optimal number of secondary users. Moreover, we prove that OR rule is superior to AND rule in detection time, and thus OR rule based CED is faster than AND rule based CED. Then, a sequential test (ST) based CED algorithm is proposed to exploit the benefit of ST and detect primary user even faster. After analyzing its detection time, we illustrate that ST based CED is able to spend the minimal detection time in satisfying the accuracy constraints by choosing an optimal sample number. Simulation results are provided to verify the effectiveness of both fast algorithms discussed in this paper.
\end{abstract}

\section{Introduction}

The significant achievement in wireless technologies has inspired masses of wireless services, which not only facilitate human life but also bring about considerable requirements on spectrum resource, causing the so-called "spectrum scarcity" problem. Cognitive radio (CR) is one of the promising techniques to alleviate this problem by allowing secondary users (SUs) to recycle the "spectrum holes" on the spectrum band that has been licensed to primary user (PU) for exclusive use. In order to identify the spectrum holes, SUs should detect whether PU is using the licensed band [1].

Several classical methods, such as energy detection, match filter detection, and cyclostationary feature detection, have been proposed for PU detection [2]. Among them, energy detection is preferable because of its simplicity, low computational complexity, and small amount of requirement on prior information about PU's signal. However, this method works badly when the signal-to-noise ratio (SNR) is low [3]. To combat the performance degradation at low SNR region, literatures $[4,5]$ suggest multiple SUs in a CR network cooperate and introduce the cooperative energy detection (CED). CED is usually implemented according to two successive stages: firstly in the sensing stage, each SU individually performs energy detection and outputs its sensing result; then in the fusing stage, a fusion center (FC) collects and fuses multiple SUs' sensing results to make a final decision. The sensing results can be either raw information SUs have observed [6-8] or local decisions SUs have made based on the observations $[9,10]$, leading to CED with soft and hard combination [11], respectively. Since the latter consumes less dedicated reporting channel resource, it increasingly becomes the dominant choice $[6,10,12]$.

Previous research on CED mainly concentrates on designing proper cooperation mechanism to achieve better detection accuracy. However, accuracy is not the only performance metric. Once PU stops using licensed band, SUs ought to immediately reuse it to increase the throughput; once PU begins occupying the band, SUs should vacate it as soon as possible to avoid causing interference $[13,14]$. 


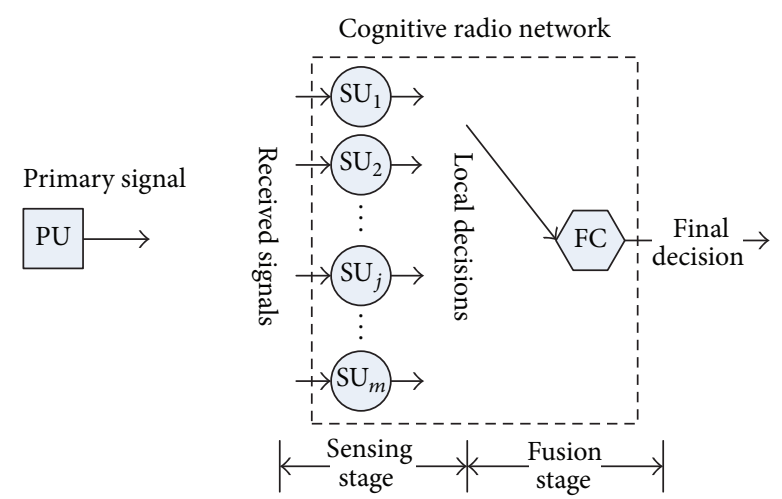

FIGURE 1: Cooperative primary user detection in CR network.

In other words, the capability of detecting PU speedily is also of vital importance. Unfortunately, there is a trade-off between detection speed and detection accuracy, and the improvement in the former usually leads to the degradation in the latter $[13,15]$.

Although CED is a popular method that has attracted great attention, its detection accuracy and detection speed are usually discussed separately. This paper jointly considers these two performance metrics based on some well-proved tools in CED and achieves fast CED via minimizing the detection time subject to the detection accuracy constraints. Fast counting rule based CED algorithm is discussed. Sequential test (ST) is applied to complete PU detection even faster. The remainder of this paper is organized as follows. Section 2 describes the CED scenario and the detection time model. Section 3 investigates the problem of minimizing detection time for counting rule based CED algorithm. Section 4 introduces the ST based CED algorithm and maximizes its detection speed. Simulation results are provided in Section 5. Section 6 concludes this paper.

\section{Scenario Description and Detection Time Model}

A CR network composed by one FC and $m$ SUs is considered in this paper. SUs implement CED with hard combination to cooperatively detect PU with the help of FC. Detailedly, the whole detection procedure comprises two stages, namely, the sensing stage and the fusion stage, as shown in Figure 1.

In the sensing stage, each SU measures the energy of its received signal, based on which a local decision is made. Assume $n$ samples are used to calculate energy, and the sampling interval is $T$. Note that calculation delay is usually negligible due to powerful computational hardware, so the time required by sensing stage can be expressed as $T_{s}=$ $n T$. Then in the fusion stage, FC collects multiple SUs' local decisions through a dedicated control channel $[6,10,12]$ and combines them to make the final decision. In order to avoid consuming too much spectrum resource, multiple SUs usually access the control channel in time division multiple access mode, and their local decisions need to be collected one by one not in parallel. Assume all $m$ SUs' local decisions are collected, and the time of collecting each SU's local decision is $R$ times of $T$. Since combination delay is also insignificant, the time required by fusion stage is given by $T_{f}=m R T$. Consequently, the detection time of CED can be modeled as [16]

$$
T_{d}=T_{s}+T_{f}=n T+m R T .
$$

This paper focuses on the detection time illustrated above and aims at minimizing it under the constraints of detection accuracy to achieve fast CED. The accuracy of final decision is usually evaluated in terms of global false alarm probability $Q_{f}$ and global missed detection probability $Q_{m}$; thus the optimization problem can be depicted as

$$
\begin{aligned}
\underset{\text { w.r.t. } m \text { or } n}{\min } & T_{d}=n T+m R T, \\
\text { s.t. } & Q_{f}=\alpha, \\
& Q_{m}=\beta,
\end{aligned}
$$

where $\alpha$ and $\beta$ represent the desired false alarm and missed probabilities, respectively.

\section{Fast Counting Rule Based CED}

As shown in Figure 1, this paper discusses CED with hard combination. One suboptimal solution to hard combination is the counting rule (also referred to as the voting rule or $\mathrm{K}$-out-of-N rule) $[4,11,12]$. This section investigates the fast counting rule based CED algorithm.

3.1. Algorithm Description. According to counting rule based $\mathrm{CED}$, each SU conducts energy detection in the sensing stage. The received energy of the $j$ th $\mathrm{SU}$ is given by

$$
v_{j}= \begin{cases}\sum_{i=1}^{n}\left|n_{j}(i)\right|^{2}, & H_{0} \\ \sum_{i=1}^{n}\left|s_{j}(i)+n_{j}(i)\right|^{2}, & H_{1}\end{cases}
$$

where $n_{j}(i)$ and $s_{j}(i)$ denote the additive noise and the received signal of the $j$ th $\mathrm{SU}$ at the $i$ th sample, respectively, and $H_{0}$ and $H_{1}$ denote the hypotheses of PU using and not using the licensed band, respectively.

Comparing $v_{j}$ with a threshold $\lambda$, the $j$ th SU can make its local decision $D_{j}$ as follows:

$$
\begin{aligned}
& v_{j}<\lambda \quad \text { Accept } H_{0} \text { and } D_{j}=0, \\
& v_{j} \geq \lambda \quad \text { Accept } H_{1} \text { and } D_{j}=1 .
\end{aligned}
$$

Without loss of generality, assuming that $n_{j}(k)$ is white and Gaussian with zero mean and unit variance and that the total energy of primary signal within each observation 
block remains constant, $v_{j}$ approximately follows Gaussian distribution according to the central limit theorem $[6,13]$ :

$$
v_{j} \sim \begin{cases}\mathcal{N}(n, 2 n), & H_{0}, \\ \mathcal{N}(n(1+\gamma), 2 n(1+2 \gamma)), & H_{1},\end{cases}
$$

where $\gamma$ is the received SNR of SU.

Based on (5), the false alarm and missed detection probabilities of SU's local decision yield to

$$
\begin{aligned}
& P_{f}=Q\left(\frac{\lambda-n}{\sqrt{2 n}}\right), \\
& P_{m}=1-Q\left(\frac{\lambda-n(1+\gamma)}{\sqrt{2 n(1+2 \gamma)}}\right),
\end{aligned}
$$

where $Q(x)=\int_{x}^{\infty}(1 / \sqrt{2 \pi}) e^{-\left(t^{2} / 2\right)} d t$ is the $Q$ function.

In the fusion stage, $\mathrm{FC}$ implements counting rule to make the final decision. More specifically, FC accepts $H_{1}$ if and only if at least $k(1 \leq k \leq m)$ SUs accept $H_{1}$; otherwise, it accepts $H_{0}$ :

$$
\begin{aligned}
& \sum_{j=1}^{m} D_{j} \geq k \quad \text { Accept } H_{1}, \\
& \sum_{j=1}^{m} D_{j}<k \quad \text { Accept } H_{0} .
\end{aligned}
$$

So the global false alarm and missed detection probabilities of the final decision can be deduced as

$$
\begin{aligned}
Q_{f} & =\sum_{j=k}^{m}\left(\begin{array}{c}
m \\
j
\end{array}\right) P_{f}^{j} \cdot\left(1-P_{f}\right)^{m-j}, \\
Q_{m} & =\sum_{j=0}^{k-1}\left(\begin{array}{c}
m \\
j
\end{array}\right)\left(1-P_{m}\right)^{j} \cdot P_{m}^{m-j} .
\end{aligned}
$$

Note that, in realistic applications, it is unnecessary for FC to firstly collect all $m$ SUs' local decisions and then count how many of them accept $H_{1}$. Once the $j$ th $(\min \{k, m-k+1\} \leq$ $j \leq m$ ) SU's decision has been collected, we may count the number of SUs accepting $H_{1}$ in $\left\{D_{1}, D_{2}, \ldots, D_{j}\right\}$ :

$$
N_{j}=\sum_{q=1}^{j} D_{q}=N_{j-1}+D_{j}
$$

where $N_{0} \triangleq 0$.

If $N_{j}$ is equal to or greater than $k, \mathrm{FC}$ could stop collecting more local decisions and make the final decision of accepting $H_{1}$ directly. If $N_{j}$ is equal to or smaller than $j-m+k+1$, FC could also stop collecting and accept $H_{0}$ because the number of SUs accepting $H_{1}$ is certainly below $k$ even though the remaining SUs all accept $H_{1}$. Otherwise, FC continues collecting the next SU's local decision $D_{j+1}$ and calculating $N_{j+1}$ :

$$
N_{j} \geq k \text { Stop collecting, accept } H_{1} \text {, }
$$

$$
N_{j} \leq j-m+k-1 \text { Stop collecting, accept } H_{0} \text {, }
$$

Otherwise Continue collecting $D_{j+1}$.

Consequently, in counting rule based CED, how many SUs' local decisions will be collected and fused to make the final decision not fixed. It is a random variable denoted by $\widehat{m}$, where $\widehat{m} \in[\min \{k, m-k+1\}, m]$.

When FC finally accepts $H_{1}$ under the hypothesis $H_{0}$, $\widehat{m}=l$ indicates that the number of SUs accepting $H_{1}$ reaches $k$ after the $l$ th SU's local decision of accepting $H_{1}$ is taken into consideration. The corresponding probability is given by

$$
\operatorname{Pr}\left(\widehat{m}=l, H_{1} \mid H_{0}\right)=P_{f} \cdot\left(\begin{array}{c}
l-1 \\
k-1
\end{array}\right) P_{f}^{k-1}\left(1-P_{f}\right)^{l-k}
$$

When FC finally accepts $H_{0}$ under the hypothesis $H_{0}, \widehat{m}=$ $l$ indicates that the number of SUs accepting $H_{0}$ reaches $m-$ $k+1$ (namely, $N_{l}$ reaches $l-m+k-1$ ) after the $l$ th SU's local decision of accepting $H_{0}$ is taken into consideration. Its probability is

$$
\begin{aligned}
\operatorname{Pr} & \left(\widehat{m}=l, H_{0} \mid H_{0}\right) \\
& =\left(1-P_{f}\right) \cdot\left(\begin{array}{c}
l-1 \\
m-k
\end{array}\right)\left(1-P_{f}\right)^{m-k} P_{f}^{l-(m-k+1)} .
\end{aligned}
$$

Similarly, we obtain

$$
\begin{aligned}
& \operatorname{Pr}\left(\widehat{m}=l, H_{1} \mid H_{1}\right) \\
& =\left(1-P_{m}\right) \cdot\left(\begin{array}{l}
l-1 \\
k-1
\end{array}\right)\left(1-P_{m}\right)^{k-1} P_{m}{ }^{l-k}, \\
& \operatorname{Pr}\left(\widehat{m}=l, H_{0} \mid H_{1}\right) \\
& \quad=P_{m} \cdot\left(\begin{array}{c}
l-1 \\
m-k
\end{array}\right) P_{m}{ }^{m-k}\left(1-P_{m}\right)^{l-(m-k+1)} .
\end{aligned}
$$

Therefore, the mean of $\widehat{m}$ can be expressed as

$$
\begin{aligned}
E\{\widehat{m}\} & \\
= & P_{0}\left[\operatorname{Pr}\left(\widehat{m}=l, H_{0} \mid H_{0}\right)+\operatorname{Pr}\left(\widehat{m}=l, H_{1} \mid H_{0}\right)\right] \\
& +P_{1}\left[\operatorname{Pr}\left(\widehat{m}=l, H_{0} \mid H_{1}\right)+\operatorname{Pr}\left(\widehat{m}=l, H_{1} \mid H_{1}\right)\right],
\end{aligned}
$$

where $P_{0}$ and $P_{1}$ are the prior probabilities of $H_{0}$ and $H_{1}$, respectively. 
Since $\widehat{m}$ is a random variable, $\widehat{T}_{d}=n T+\widehat{m} R T$ is also a random variable. As a result, the optimization problem described in (2) should be rewritten as

$$
\begin{array}{rl}
\min _{\text {w.r.t. } m \text { or } n} & E\left\{\widehat{T}_{d}\right\}=n T+E\{\widehat{m}\} R T \\
\text { s.t. } & Q_{f}=\alpha, \\
& Q_{m}=\beta .
\end{array}
$$

In the rest of this paper, we will discuss (15) instead of (2).

3.2. $k=m$ (AND Rule). Considering the counting rule with parameter $k(1 \leq k \leq m)$, this subsection deals with a special case of $k=m$ for simplicity. In this case, FC accepts $H_{1}$ if and only if all SUs accept $H_{1}$, and the counting rule turns to be the "AND rule" [2].

Substituting $k=m$ into (8), the constraints on detection accuracy can be reexpressed as

$$
\begin{aligned}
& Q_{f}=P_{f}^{m}=\alpha, \\
& Q_{m}=1-\left(1-P_{m}\right)^{m}=\beta .
\end{aligned}
$$

In addition, using the results of (6) to deduce $n$ and $\lambda$, we get

$$
\begin{aligned}
n= & \frac{2}{\gamma^{2}}\left[Q^{-1}(\sqrt[m]{\alpha})-\sqrt{1+2 \gamma} Q^{-1}(\sqrt[m]{1-\beta})\right]^{2}, \\
\lambda= & \frac{2}{\gamma^{2}}\left\{(1+\gamma)\left[Q^{-1}(\sqrt[m]{\alpha})\right]^{2}\right. \\
& -(2+\gamma) \sqrt{1+2 \gamma} Q^{-1}(\sqrt[m]{\alpha}) Q^{-1}(\sqrt[m]{1-\beta}) \\
& \left.+(1+2 \gamma)\left[Q^{-1}(\sqrt[m]{1-\beta})\right]^{2}\right\} .
\end{aligned}
$$

It should be pointed out that, given a number of SUs $m$, accuracy constraints can be satisfied if the sample number $n$ and the threshold $\lambda$ are chosen according to (17) and (18).

Substituting $k=m$ and (16) into (14), the mean of $\widehat{m}$ yields to

$$
E\{\widehat{m}\}=P_{0} \frac{1-\alpha}{1-\sqrt[m]{\alpha}}+P_{1} \frac{\beta}{1-\sqrt[m]{1-\beta}} .
$$

Finally, the mean of detection time for AND rule based CED is given by

$$
\begin{aligned}
E\left\{\widehat{T}_{d}\right\}= & \frac{2 T}{\gamma^{2}}\left[Q^{-1}(\sqrt[m]{\alpha})-\sqrt{1+2 \gamma} Q^{-1}(\sqrt[m]{1-\beta})\right]^{2} \\
& +R T\left(P_{0} \frac{1-\alpha}{1-\sqrt[m]{\alpha}}+P_{1} \frac{\beta}{1-\sqrt[m]{1-\beta}}\right)
\end{aligned}
$$

Note that both $\alpha$ and $\beta$ are usually small, and thus the left part of $E\left\{\widehat{T}_{d}\right\}$ decreases as $m$ increases, while its right part increases as $m$ increases. Intuitively, neither too many nor too few SUs should be used. There exists an optimal number of SUs with which the average detection time can be minimized.
Since $m$ is a natural number, its optimal value $m_{\mathrm{opt}}$ can be easily obtained via numerical search according to (20). Substituting $m_{\mathrm{opt}}$ into (17) and (18), the optimal values for the sample number and the threshold, namely, $n_{\text {opt }}$ and $\lambda_{\text {opt }}$, can be derived as well. In practical scenarios, if implementing AND rule based CED according to the parameters $m_{\text {opt }}, n_{\text {opt }}$, and $\lambda_{\text {opt }}$, we will not only guarantee the desired detection accuracy but also minimize the detection time. In other words, the expected fast PU detection is achieved.

3.3. $k=1$ (OR Rule). Besides $k=m$, another special case for the counting rule with parameter $k(1 \leq k \leq m)$ is $k=1$; that is, FC accepts $H_{1}$ if any one of $m$ SUs accepts $H_{1}$, resulting in the so-called "OR rule" [2].

Substituting $k=1$ into (8) and (14), we obtain

$$
\begin{aligned}
Q_{f} & =1-\left(1-P_{f}\right)^{m}=\alpha, \\
Q_{m} & =P_{m}^{m}=\beta, \\
E\{\widehat{m}\} & =P_{0} \frac{\alpha}{1-\sqrt[m]{1-\alpha}}+P_{1} \frac{1-\beta}{1-\sqrt[m]{\beta}} .
\end{aligned}
$$

Using the equations above, the sample number $n$, the threshold $\lambda$, and the average detection time $E\left\{\widehat{T}_{d}\right\}$ of OR rule based CED are given by

$$
\begin{aligned}
& n= \frac{2}{\gamma^{2}}\left[-Q^{-1}(\sqrt[m]{1-\alpha})+\sqrt{1+2 \gamma} Q^{-1}(\sqrt[m]{\beta})\right]^{2}, \\
& \lambda=\frac{2}{\gamma^{2}}\left\{(1+\gamma)\left[Q^{-1}(\sqrt[m]{1-\alpha})\right]^{2}\right. \\
&-(2+\gamma) \sqrt{1+2 \gamma} Q^{-1}(\sqrt[m]{1-\alpha}) Q^{-1}(\sqrt[m]{\beta}) \\
&\left.+(1+2 \gamma)\left[Q^{-1}(\sqrt[m]{\beta})\right]^{2}\right\}, \\
& E\left\{\widehat{T}_{d}\right\}=\frac{2 T}{\gamma^{2}}\left[-Q^{-1}(\sqrt[m]{1-\alpha})\right. \\
&\left.+\sqrt{1+2 \gamma} Q^{-1}(\sqrt[m]{\beta})\right]^{2}+R T\left(P_{0} \frac{\alpha}{1-\sqrt[m]{1-\alpha}}\right. \\
&\left.+P_{1} \frac{1-\beta}{1-\sqrt[m]{\beta}}\right) \cdot
\end{aligned}
$$

Similarly, the optimal value for the number of SUs to minimize $E\left\{\widehat{T}_{d}\right\}$, namely, $m_{\text {opt }}$, can be obtained via numerical search according to (24). $n_{\text {opt }}$ and $\lambda_{\text {opt }}$ can be derived from (22) and (23), respectively. With these parameters, fast OR rule based CED will be achieved in terms of minimizing average detection time subject to desired detection accuracy.

Moreover, in order to compare two special cases of $k=m$ and $k=1$, we define the average detection time difference as

$$
\Delta T_{d}=E\left\{\widehat{T}_{d} \mid k=1\right\}-E\left\{\widehat{T}_{d} \mid k=m\right\} .
$$


Without loss of generality, considering $P_{0}=P_{1}$ and $\alpha=$ $\beta<1 / 2$, it is easy to deduce

$$
\Delta T_{K M}=\frac{4 T}{\gamma}\left\{\left[Q^{-1}(\sqrt[m]{\alpha})\right]^{2}-\left[Q^{-1}(\sqrt[m]{1-\alpha})\right]^{2}\right\}<0
$$

Therefore, given the same number of SUs and the same accuracy constraints, OR rule consumes less detection time compared with AND rule. In other words, it is faster than AND rule.

\section{Fast ST Based CED}

4.1. Algorithm Description. Invented by Wald, ST is superior in consuming less observations to control both false alarm and missed detection errors compared with other traditional test methods $[15,17]$. In this section, FC implements ST to fuse multiple SUs' local decisions, resulting in the ST based CED.

The sensing stage of ST based CED is identical with that of counting rule based CED. In the fusion stage, FC collects multiple SUs' local decisions one by one and fuses them to make the final decision according to ST instead of counting rule. When the $j$ th local decision $D_{j}$ is obtained, its loglikelihood ratio $L D_{j}$ and cumulative log-likelihood ratio $S D_{j}$ can be derived as

$$
\begin{aligned}
L D_{j} & =\log \left(\frac{\operatorname{Pr}\left(D_{j} \mid H_{1}\right)}{\operatorname{Pr}\left(D_{j} \mid H_{0}\right)}\right) \\
& =\left\{\begin{array}{l}
\log \left(\frac{P_{m}}{1-P_{f}}\right) \quad D_{j}=0 \\
\log \left(\frac{1-P_{m}}{P_{f}}\right) \quad D_{j}=1,
\end{array}\right. \\
S D_{j} & =\sum_{q=1}^{j} L D_{q}=S D_{j-1}+L D_{j},
\end{aligned}
$$

where $S D_{0} \triangleq 0$

Comparing $S D_{j}$ with two thresholds $\lambda_{L}$ and $\lambda_{H}\left(\lambda_{L}<\right.$ $\lambda_{H}$ ), whether making the final decision or continuing collecting the next SU's local decision is determined as follows:

$$
\begin{aligned}
& S D_{j} \geq \lambda_{H} \quad \text { Accept } H_{1}, \\
& S D_{j} \leq \lambda_{L} \quad \text { Accept } H_{0}, \\
& \lambda_{L}<S D_{j}<\lambda_{H} \quad \text { Continue collecting } D_{j+1},
\end{aligned}
$$

where both $\lambda_{L}$ and $\lambda_{H}$ depend on the desired detection accuracy $\alpha$ and $\beta$. According to the propositions in [17], these thresholds are given by $\lambda_{L}=\log (\beta /(1-\alpha)), \lambda_{H}=\log ((1-$ $\beta) / \alpha)(\alpha<1 / 2, \beta<1 / 2)$

4.2. Detection Time Optimization. Inherited from the feature of ST, ST based CED is able to stop collecting once the collected local decisions are sufficient enough to guarantee the desired accuracy. It has two distinctive characteristics: (1) accuracy requirements are autonomously satisfied via ST as shown in (28), so there is no need to care about its detection accuracy any more. (2) How many SUs' local decisions will be collected and fused before making the final decision is not fixed. Similarly, denote this random variable by $\widehat{m}$. The means of $\widehat{m}$ under both hypotheses are given by [17]

$$
\begin{aligned}
& E\left\{\widehat{m} \mid H_{0}\right\} \\
& \approx \frac{(1-\alpha) \log (\beta /(1-\alpha))+\alpha \log ((1-\beta) / \alpha)}{E\left\{L D_{j} \mid H_{0}\right\}} \\
& =\frac{(1-\alpha) \log (\beta /(1-\alpha))+\alpha \log ((1-\beta) / \alpha)}{\left(1-P_{f}\right) \log \left(P_{m} /\left(1-P_{f}\right)\right)+P_{f} \log \left(\left(1-P_{m}\right) / P_{f}\right)}, \\
& E\left\{\widehat{m} \mid H_{1}\right\} \\
& \approx \frac{\beta \log (\beta /(1-\alpha))+(1-\beta) \log ((1-\beta) / \alpha)}{E\left\{L D_{j} \mid H_{1}\right\}} \\
& =\frac{\beta \log (\beta /(1-\alpha))+(1-\beta) \log ((1-\beta) / \alpha)}{P_{m} \log \left(P_{m} /\left(1-P_{f}\right)\right)+\left(1-P_{m}\right) \log \left(\left(1-P_{m}\right) / P_{f}\right)} .
\end{aligned}
$$
to

Then the average detection time of ST based CED yields

$$
E\left\{\widehat{T}_{d}\right\}=n T+\left(P_{0} E\left\{\widehat{m} \mid H_{0}\right\}+P_{1} E\left\{\widehat{m} \mid H_{1}\right\}\right) R T .
$$

As shown in (30), $E\left\{\widehat{T}_{d}\right\}$ depends on the variables $n, P_{f}$, and $P_{m}$. Because of (6), $E\left\{\widehat{T}_{d}\right\}$ is eventually determined by the parameters $n$ and $\lambda$. Conducting two-dimensional numerical search based on (30), a pair of optimal values for $n$ and $\lambda$ can be obtained to minimize $E\left\{\widehat{T}_{d}\right\}$ and achieve fast ST based CED.

Without loss of generality, we assume $\alpha=\beta$ and $P_{f}=P_{m}$ to balance false alarm or missed detection errors. As a result, $\lambda$ can be written as a function of $n$ :

$$
\lambda=\frac{\sqrt{1+2 \gamma}+1+\gamma}{\sqrt{1+2 \gamma}+1} n
$$

and $E\left\{\widehat{T}_{d}\right\}$ solely depends on $n$ :

$$
E\left\{\widehat{T}_{d}\right\}=n T+\frac{(1-2 \alpha) \log ((1-\alpha) / \alpha) R T}{[1-2 Q(\sqrt{2 n} \cdot \gamma / 2(\sqrt{1+2 \gamma}+1))] \log (1 / Q(\sqrt{2 n} \cdot \gamma / 2(\sqrt{1+2 \gamma}+1))-1)}
$$




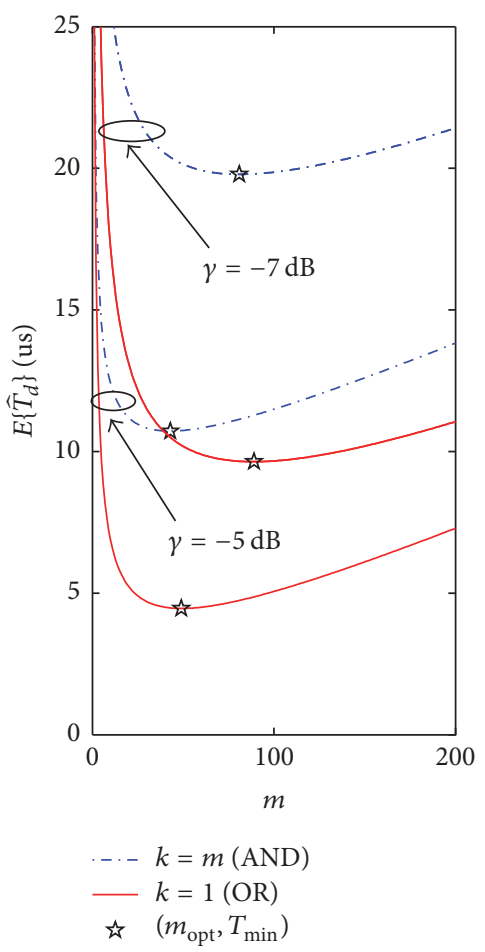

(a)

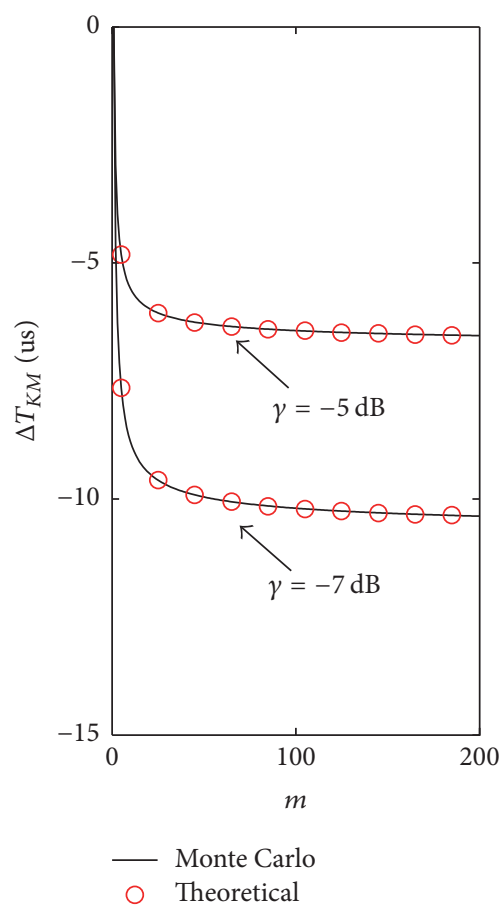

(b)

FIGURE 2: Average detection time and detection time difference of counting rule based CED versus SU number for $\gamma=-5,-7 \mathrm{~dB}$.

The optimal sample number to minimize $E\left\{\widehat{T}_{d}\right\}$, namely, $n_{\mathrm{opt}}$, can be easily derived from (32), with which the speed of ST based CED is consequently maximized.

\section{Numerical Results}

This section provides some simulation results to verify the theoretical derivations above. Simulation settings are listed as follows: $T=0.046459$ us [18], $R=1, \alpha=\beta=1 \%$, and $P_{0}=P_{1}$.

In order to test the detection accuracy of fast counting rule based CED, Monte Carlo simulations are conducted with different numbers of SUs $(m=10,50,100,150,200)$ for $\gamma=-5 \mathrm{~dB}$. Given a specific $m$, if $k=m$, parameters $n$ and $\lambda$ are chosen according to (17) and (18), respectively, and the accuracy results $\left(Q_{f}\right.$ and $\left.Q_{m}\right)$ are recorded in Table 1 . If $k=1$, parameters $n$ and $\lambda$ are derived from (22) and (23), respectively, and the corresponding results are demonstrated in Table 2. It can be seen from both tables that, no matter how $m$ changes, the global false alarm and missed detection probabilities always remain constant and practically equal $1 \%$. Therefore, the proposed fast counting rule based CED is able to meet the accuracy constraints.

Since the accuracy constraints have been satisfied, we focus on the performance metric of detection time in Figure 2. Considering $k=m$ and $k=1$, Figure 2(a) plots the average detection time curves of counting rule based CED versus the number of SUs for $\gamma=-5,-7 \mathrm{~dB}$. As shown in this subfigure, all curves are concave regardless of $k$ and $\gamma$. In other words, there exists an optimal number of SUs to minimize
TABLE 1: Detection accuracy of counting rule based CED with $k=m$ for $\gamma=-5 \mathrm{~dB}$.

\begin{tabular}{lcccc}
\hline$m$ & $n$ & $\lambda$ & $Q_{f}$ & $Q_{m}$ \\
\hline 10 & 261 & 253.32 & $0.99 \%$ & $0.99 \%$ \\
50 & 201 & 173.63 & $0.95 \%$ & $1.00 \%$ \\
100 & 187 & 153.88 & $1.02 \%$ & $0.95 \%$ \\
150 & 180 & 144.60 & $1.02 \%$ & $1.03 \%$ \\
200 & 176 & 138.81 & $1.02 \%$ & $1.02 \%$ \\
\hline
\end{tabular}

TABLE 2: Detection accuracy of counting rule based CED with $k=1$ for $\gamma=-5 \mathrm{~dB}$.

\begin{tabular}{lcccc}
\hline$m$ & $n$ & $\lambda$ & $Q_{f}$ & $Q_{m}$ \\
\hline 10 & 142 & 193.68 & $0.97 \%$ & $1.00 \%$ \\
50 & 66 & 106.01 & $1.02 \%$ & $0.96 \%$ \\
100 & 48 & 84.64 & $0.98 \%$ & $0.99 \%$ \\
150 & 40 & 74.65 & $1.03 \%$ & $0.97 \%$ \\
200 & 36 & 68.43 & $0.97 \%$ & $0.97 \%$ \\
\hline
\end{tabular}

the average detection time. Implementing numerical search according to (20) and (24), the theoretical optimal number of SUs $m_{\mathrm{opt}}$ and the minimal average detection time $T_{\min }$ are obtained and plotted. Note that each point of $\left(m_{\mathrm{opt}}, T_{\min }\right)$ agrees well with the bottom of corresponding detection time curve. This phenomenon shows the effectiveness of our method to achieve the highest detection speed. Moreover, to dig into the special cases of $k=m$ and $k=1$, Figure 2(b) 
TABLE 3: Detection accuracy of ST based CED for $\gamma=-5 \mathrm{~dB}$.

\begin{tabular}{cccc}
\hline$n$ & $\lambda$ & $Q_{f}$ & $Q_{m}$ \\
\hline \multirow{3}{*}{50} & 46.94 & $0.82 \%$ & $0.62 \%$ \\
& $56.94\left(P_{f}=P_{m}\right)$ & $0.32 \%$ & $0.33 \%$ \\
& 66.94 & $0.56 \%$ & $0.90 \%$ \\
& 103.88 & $0.61 \%$ & $0.30 \%$ \\
100 & $113.88\left(P_{f}=P_{m}\right)$ & $0.76 \%$ & $0.75 \%$ \\
& 123.88 & $0.35 \%$ & $0.61 \%$ \\
\hline
\end{tabular}

depicts their average detection time difference versus the number of SUs for $\gamma=-5,-7 \mathrm{~dB}$. In this subfigure, the Monte Carlo curves are derived from Monte Carlo simulations, and the theoretical curves are calculated by (26). As two types of curves are overlapping and always below 0 , the correctness of (26) is verified. So the average detection time of counting based CED with $k=1$ is smaller than that with $k=m$. In other words, OR rule based CED is faster than AND rule based CED.

Aiming to verify the accuracy of fast ST based CED, we perform Monte Carlo simulations with various sample numbers $(n=50,100)$ for $\gamma=-5 \mathrm{~dB}$, and record the global false alarm as well as missed detection probabilities in Table 3. Given each $n$, three values of the local threshold $\lambda$ are involved: the value calculated by (31) to guarantee $P_{f}=P_{m}$ $(\lambda=56.94,113.88)$, and the calculated value with \pm 10 offsets. As shown in Table 3, both $Q_{f}$ and $Q_{m}$ are always smaller than $1 \%$, indicating that the proposed fast ST based CED satisfies the accuracy constraints well.

Finally, Figure 3 plots the average detection time curves of counting rule based CED and ST based CED versus the sample number for $\gamma=-5 \mathrm{~dB}$. In counting rule based CED, both $k=m$ and $k=1$ are investigated. As shown in this figure, the curve of $k=1$ is fairly lower, and thus the superiority of OR rule over AND rule is verified again. In ST based CED, $P_{f}=P_{m}$ is considered, the Monte Carlo curve is obtained by Monte Carlo simulation, and the theoretical curve is calculated by (32). Three phenomena should be emphasized here: (1) Monte Carlo and theoretical curves agree well with each other, which demonstrates the validity of (32); (2) the curves of ST based CED are even lower than that of counting rule based CED with $k=1$, which shows that ST based CED is faster; (3) the ST curves are concave, and thereby it is possible to find an optimal sample number to minimize the average detection time. Furthermore, we perform numerical search according to (32) and derive the optimal sample number $n_{\text {opt }}$ as well as the corresponding minimal detection time $T_{\min }$. Note that the point of $\left(n_{\mathrm{opt}}, T_{\mathrm{min}}\right)$ is located at the bottom of Monte Carlo curve. Therefore, by properly selecting a sample number as suggested, the proposed ST based CED is able to minimize the average detection time and achieve the highest detection speed.

\section{Conclusion}

This paper has jointly considered two performance metrics of CED and realized fast CED via minimizing the detection time under the constraints on detection accuracy. Some

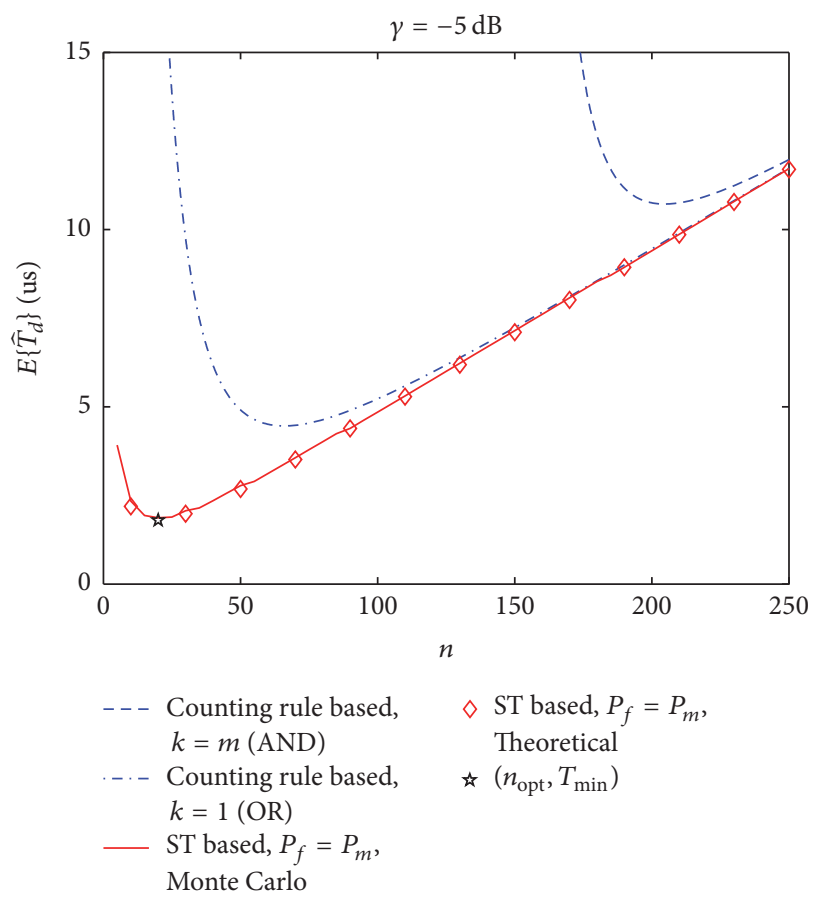

Figure 3: Average detection time of counting rule and ST based CED versus sample number for $\gamma=-5 \mathrm{~dB}$.

well-proved tools of CED were employed. The counting rule based CED algorithm was discussed. Its detection accuracy and detection time were analyzed. Considering the special cases of counting rule (AND rule and OR rule), we illustrated that the minimal detection time could be achieved by appropriately choosing the number of SUs. Moreover, we proved that, under the same accuracy constraints, $O R$ rule consumed less detection time and was faster than AND rule. Then, by replacing counting rule with ST, the ST based CED algorithm was proposed. This algorithm inherited the benefit of ST and was able to complete PU detection even faster. Its detection time was also analyzed. Via minimizing the detection time, an optimal sample number was deduced, with which ST based CED could obtain the highest detection speed while guaranteeing the desired detection accuracy.

It should be pointed out that although this paper merely illustrates how to combine the detection results of multiple SUs in CR networks, our approach can be applied to the multiantenna scenarios, and how to appropriately combine the outputs of multiple antennas to achieve fast detection is also worth investigating.

Moreover, since the computational complexity of CED also depends on the sample number and the number of SUs, it can be similarly modeled as in (1). Consequently, the performance of computational complexity is similar to that of detection time; that is, ST based CED is the best, and AND rule based CED is the worst.

\section{Conflicts of Interest}

The authors declare that there are no conflicts of interest regarding the publication of this paper. 


\section{Acknowledgments}

This research is supported by the National Natural Science Foundation of China (nos. 61201264, 61362018), China Scholarship Council (no. 201508350023), Fundamental Research Funds for the Central Universities (no. 2242015R20003), Jiangsu Province Postdoctoral Scientific Research Project of China (no. 1402041B), and Huaqiao University (no. 13BS101).

\section{References}

[1] S. Filin, T. Baykas, H. Harada, F. Kojima, and H. Yano, "IEEE Standard 802.19.1 for TV white space coexis tence," IEEE Communications Magazine, vol. 54, no. 3, pp. 22-26, 2016.

[2] I. F. Akyildiz, W. Lee, M. C. Vuran, and S. Mohanty, "NeXt generation/dynamic spectrum access/cognitive radio wireless networks: a survey," Computer Networks, vol. 50, no. 13, pp. 21272159, 2006.

[3] R. Tandra and A. Sahai, "SNR walls for signal detection," IEEE Journal on Selected Topics in Signal Processing, vol. 2, no. 1, pp. 4-17, 2008.

[4] J. Unnikrishnan and V. V. Veeravalli, "Cooperative sensing for primary detection in cognitive radio," IEEE Journal on Selected Topics in Signal Processing, vol. 2, no. 1, pp. 18-27, 2008.

[5] I. F. Akyildiz, B. F. Lo, and R. Balakrishnan, "Cooperative spectrum sensing in cognitive radio networks: a survey," Physical Communication, vol. 4, no. 1, pp. 40-62, 2011.

[6] J. Ma, G. Zhao, and Y. Li, "Soft combination and detection for cooperative spectrum sensing in cognitive radio networks," IEEE Transactions on Wireless Communications, vol. 7, no. 11, pp. 4502-4507, 2008.

[7] W. Han, J. Li, Z. Li, J. Si, and Y. Zhang, "Efficient soft decision fusion rule in cooperative spectrum sensing," IEEE Transactions on Signal Processing, vol. 61, no. 8, pp. 1931-1943, 2013.

[8] W. Choi, M.-G. Song, J. Ahn, and G.-H. Im, "Soft combining for cooperative spectrum sensing over fast-fading channels," IEEE Communications Letters, vol. 18, no. 2, pp. 193-196, 2014.

[9] W. Zhang, R. K. Mallik, and K. B. Letaief, "Cooperative spectrum sensing optimization in cognitive radio networks," in Proceedings of the IEEE International Conference on Communications (ICC '08), pp. 3411-3415, May 2008.

[10] Y. Zou, Y.-D. Yao, and B. Zheng, "A selective-relay based cooperative spectrum sensing scheme without dedicated reporting channels in cognitive radio networks," IEEE Transactions on Wireless Communications, vol. 10, no. 4, pp. 1188-1198, 2011.

[11] S. Atapattu, C. Tellambura, and H. Jiang, "Energy detection based cooperative spectrum sensing in cognitive radio networks," IEEE Transactions on Wireless Communications, vol. 10, no. 4, pp. 1232-1241, 2011.

[12] A. Jamshidi, "Performance analysis of low average reporting bits cognitive radio schemes in bandwidth constraint control channels," IET Communications, vol. 3, no. 9, pp. 1544-1556, 2009.

[13] Y.-C. Liang, Y. Zeng, E. Peh, and A. T. Hoang, "Sensingthroughput tradeoff for cognitive radio networks," IEEE Transactions on Wireless Communications, vol. 7, no. 4, pp. 1326-1337, 2008.

[14] Y. Zou, Y.-D. Yao, and B. Zheng, "Outage probability analysis of cognitive transmissions: Impact of spectrum sensing overhead," IEEE Transactions on Wireless Communications, vol. 9, no. 8, pp. 2676-2688, 2010.
[15] Q. Zou, S. Zheng, and A. H. Sayed, "Cooperative sensing via sequential detection," IEEE Transactions on Signal Processing, vol. 58, no. 12, pp. 6266-6283, 2010.

[16] S. Peng, X. Yang, S. Shu, P. Zhu, and X. Cao, "Adaptive sequential cooperative energy detection scheme for primary user detection in cognitive radio," IEICE Transactions on Communications, vol. E94-B, no. 10, pp. 2896-2899, 2011.

[17] A. Wald, "Sequential tests of statistical hypotheses," Annals of Mathematical Statistics, vol. 16, no. 2, pp. 117-186, 1945.

[18] Y. Zeng and Y.-C. Liang, "Covariance based signal detections for cognitive radio," in Proceedings of the 2nd IEEE International Symposium on New Frontiers in Dynamic Spectrum Access Networks, pp. 202-207, IEEE, Dublin, Ireland, April 2007. 


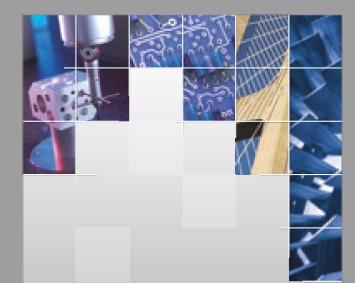

\section{Enfincering}
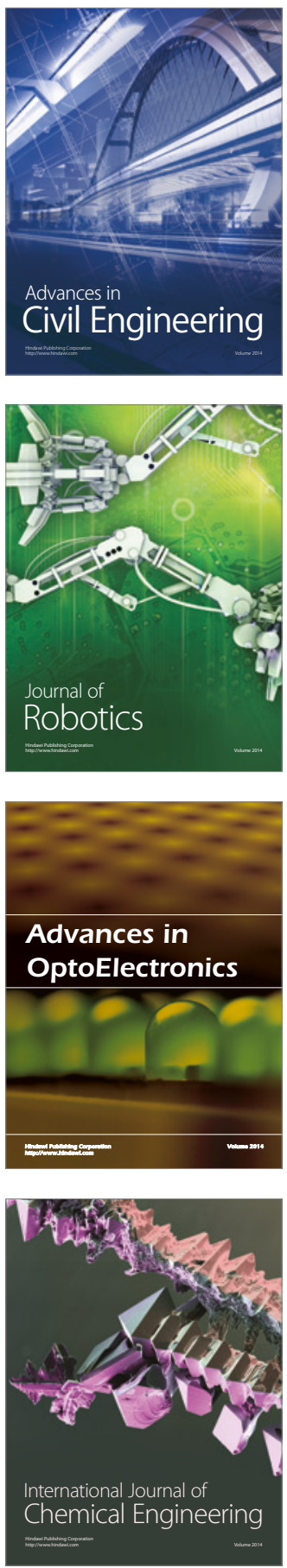

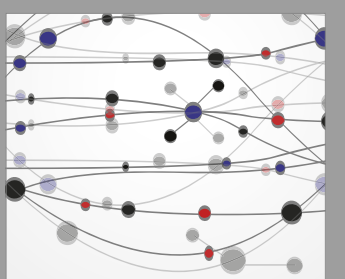

The Scientific World Journal

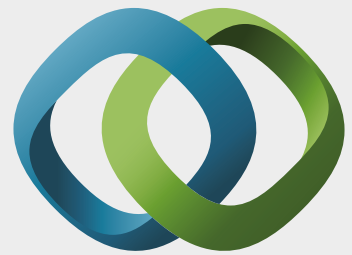

\section{Hindawi}

Submit your manuscripts at

https://www.hindawi.com
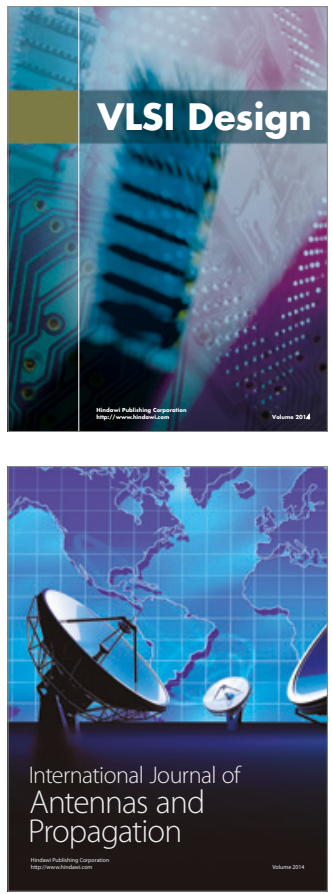

\section{Rotating}

Machinery
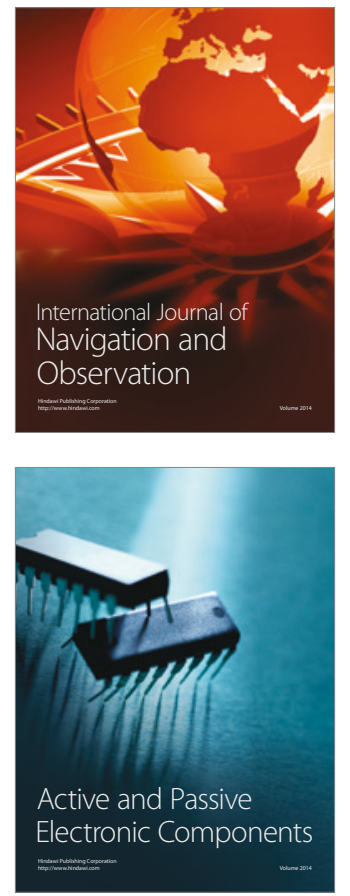
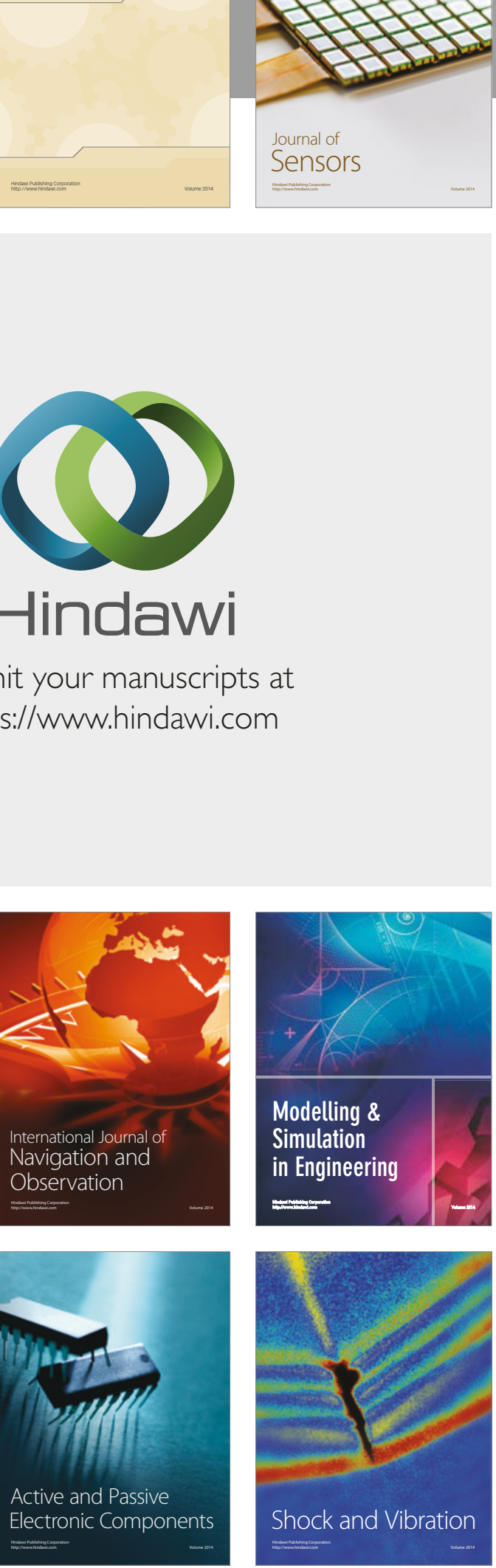
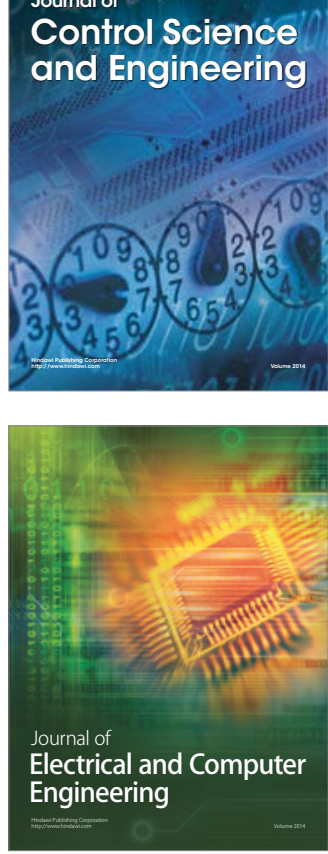

Distributed

Journal of

Control Science

and Engineering
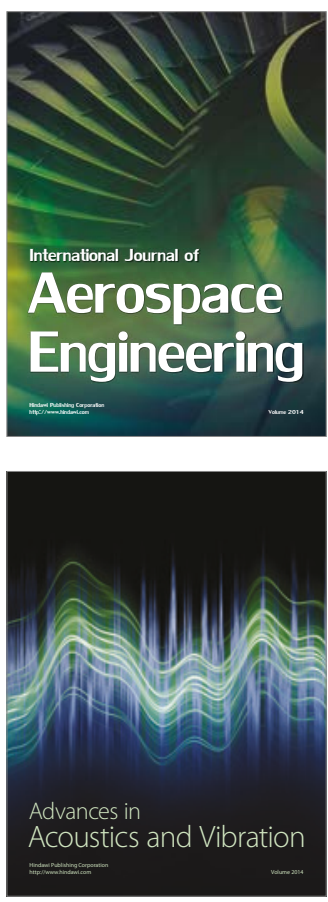

Sensor Networks 